See discussions, stats, and author profiles for this publication at: https://www.researchgate.net/publication/330391035

\title{
Engineering an Aligned Gold-Standard Corpus of Human to Machine Oriented Controlled Natural Language
}

Conference Paper · December 2018

DOI: $10.1109 /$ WI.2018.00-58

CITATION

1

3 authors:

Hazem Safwat

National University of Ireland, Galway

6 PUBLICATIONS 19 CITATIONS

SEE PROFILE

0

Manel Zarrouk

National University of Ireland, Galway

37 PUBLICATIONS 146 CITATIONS

SEE PROFILE

Some of the authors of this publication are also working on these related projects:

SSIX Project View project

Discourse Analysis for Automatic Fake News Classification View project
READS

21

Brian Davis
National University of Ireland, Galway
68 PUBLICATIONS 625 CITATIONS
SEE PROFILE




\section{Engineering an aligned gold-standard corpus of human to machine oriented Controlled Natural Language}

\author{
Hazem Safwat \\ Insight Centre for Data Analytics \\ National University of Ireland, Galway \\ hazem.abdelaal@insight-centre.org
}

\author{
Brian Davis \\ Insight Centre for Data Analytics \\ National University of Ireland, Galway \\ brian.davis@insight-centre.org
}

\author{
Manel Zarrouk \\ Insight Centre for Data Analytics \\ National University of Ireland, Galway \\ manel.zarrouk@insight-centre.org
}

\begin{abstract}
Knowledge base creation and population are an essential formal backbone for a variety of intelligent applications, decision support and expert systems and intelligent search. While the abundance of unstructured text helps in easing the knowledge acquisition gap, the ambiguous nature of language tends to impact accuracy when engaging in more complex semantic analysis. Controlled Natural Languages (CNLs) are subsets of natural language that are restricted grammatically in order to reduce or eliminate ambiguity for the purposes of machine processability, or unambiguous human communication within a domain or industry context, such as Simplified English. This type of human-oriented CNL is under-researched despite having found favor within industry over many years. We describe a novel dataset which aligns a representative sample of Simplified English Wikipedia sentences with a well known machine-oriented CNL. This linguistic resource is both human-readable and semantically machine interpretable and can benefit a variety of NLP and knowledge based applications.

Index Terms-Natural Language Processing, Controlled Natural Language, Knowledge Extraction, Semantic Web
\end{abstract}

\section{INTRODUCTION}

Knowledge base creation and population is of paramount importance for a variety of applications including decision support and expert systems. Formal knowledge may also act as semantic backbone for language processing and information retrieval applications. A knowledge base for a particular domain may be either non existent or incomplete. While the abundance of unstructured text offers a means of easing the knowledge acquisition gap, the highly ambiguous nature of language impacts on accuracy when moving beyond the entity level to more complex semantic interpretation of text for knowledge creation. Controlled Natural Languages (CNLs) are unambiguous natural languages based on a restricted grammar that map into formal knowledge structures [1]. CNLs are an attempt to circumvent linguistic ambiguity and have found applications with respect to formal knowledge creation, ontology development and domain specific knowledge based machine translation [2]. Aside from specific knowledge gathering activities, which by the vary nature of the task, demand a restricted natural language interface for knowledge creation by domain experts, CNLs offer little incentive to the average user to create formal knowledge, even as implicit side effect of an authoring effort involving semantic annotation or enrichment of text content. A subcategory of CNL which offers a middle ground of reduced ambiguity for semantic interpretation but less restriction than a machine- oriented CNL. Human-oriented CNLs [3] have been in wide spread use for many years. Their development was motivated by the purposes of language learning, and unambiguous communication between humans in a domain context. An example of human-oriented CNL is the Simplified text such as Simple Wikipedia ${ }^{1}$. It is a form of text written using style guides ${ }^{2}$ to reduce complexity and ambiguity of the language, especially for non-native speakers and juniors. Some of the written style guides recommended for authors in Simple Wikipedia include: use active voices, avoid compound sentences(e.g conjunctions), avoid idioms (multiwords), keep sentences short and informative. Other humanoriented CNLs include ASD Simplified Technical English ${ }^{3}$, developed to improve the readability and comprehensibility in technical documents. In addition to, Boeing Technical English to improve the communication between people for air traffic control [4]. The development and planning of these CNLs appears often community driven, many of which at first glance, like Simplified Wikipedia, may been inspired by Basic English [5]. Human-oriented CNLs unlike machine-oriented CNLs [3] do not attempt to unambiguously map into formal knowledge structures, as the communication goal is human to human and not human to machine. An interesting premise is how close linguistically such languages are to machine-oriented CNLs and if so to what degree? Moreover, rewriting all or most of a human-oriented CNLs into a machine-oriented CNL which can be unambiguously parsed into a formal knowledge structure [17] could unlock significant silos of implicit general purpose domain knowledge, contained within existing human-oriented CNL content.

This paper focuses on our initial experiments with respect to the computational linguistic analysis of a corpus of humanoriented CNL represented in Simplified English, as well as the

\footnotetext{
${ }^{1}$ https://simple.wikipedia.org/wiki/

${ }^{2} \mathrm{https} / / /$ simple.wikipedia.org/wiki/Wikipedia:How_to_write_Simple_ English_pages

${ }^{3}$ http://www.asd-ste100.org/
} 
investigation of the feasibility of rewriting Simplified English into a well know machine-oriented CNL called ACE [7]. Since no ground truth exists, it has been necessary to engineer one for our experiments.

We present a linguistic resource that is both human-readable and semantically machine interpretable. This resource is a snapshot taken from the abstracts of the Simple English Wikipedia dump ${ }^{4}$. The selected abstracts are rewritten into a machine-oriented CNL, by applying some rules on the syntactic structures of the sentences to be accepted and parsed by the CNL semantic parser. To our knowledge this resource is the first human to machine CNL aligned dataset. The paper is structured as follows: Section II describes related work, Section III presents the corpus collection, processing and analysis. In Section IV, we discuss the results and the evaluation, and finally, Section V offers a conclusion.

\section{RELATED WORK}

Attempto Controlled English (ACE) is a CNL developed for knowledge representation. We choose ACE for our experiments for the following reasons: 1) It is a widely adopted CNL with an expressive grammar. 2) The language can be automatically mapped into different formal languages such as Discourse Representation Structures (variant of first order logic) [7] and subsequently a subset of ACE can be converted to the Web Ontology Language (OWL). 3) It also provides access to different tools and resources to use the language such as the ACE parsing engine - $\mathrm{APE}^{5}$, which we use for our validation.

The work from [8] analysed the text in Simple Wikipedia and Wikipedia linguistic features to produce a new resource corpus that can help sentence simplification research. It provides a new simplification dataset that is an improved version over Simple Wikipedia. Their analysis over the text in both Simple Wikipedia and Wikipedia was done for the purpose of showing the amount of simplification between two parallel sentences from both Wikipedias, and introduce a new comparative approach to simplification corpus analysis. The main difference between our work is that we analyse both Wikipedias for the purpose of rewriting simplified English into a formal knowledge using CNLs as they tend to be less ambiguous.

In [9] the authors present a text rewriting approach to increase the amount of labeled data available for model training. They analysed Simple Wikipedia and Wikipedia parallel corpora to automatically extract rewrite rules, then generate multiple versions of sentences annotated with gold standard labels for the purposes of semantic role labeling. Our work is similar to them in that we also use rewrite rules to generate gold standard resource. However, the main difference is that we use rewrite rules to generate CNL sentences from Simple Wikipedia sentences, which means our efforts are directed towards a different purpose.

\footnotetext{
${ }^{4}$ https://dumps.wikimedia.org/simplewiki/

${ }^{5}$ https://github.com/Attempto/APE
}

In [10] the author, presents a semantically annotated corpus developed using a bootstrapping approach using NLP techniques for parsing and semantic interpretation, together with an interface for collaborative annotation of experts and crowdsourcing community. Although they generated a semantic resource with deep semantics, the resource is small as it includes less than $5 \mathrm{k}$ sentences which is much smaller than ours.

The other resources of ProPBank [11] and Framenet [12] are semantically annotated corpora. Although, both of the resources are valuable to the community, they lack the deep semantic representation that combines different layers of semantic annotation into one formalism [10].

\section{CORPUS ANALYSIS}

We first collected a dataset of the abstracts from the Simple Wikipedia dump and its parallel Wikipedia dump. All experiments are performed on Simple Wikipedia abstracts only and their corresponding Wikipedia abstracts. Table I, describes the style guides from Simple Wikipedia on how to write simplified text versus several common stylistic properties observed across several CNLs (both human and machine oriented) [13]. From the table we can see that both texts significantly overlap in most of the properties.

\section{A. Corpus Collection and Pre-processing}

We collected the XML formatted dumps containing both Simple Wikipedia abstracts and their corresponding Wikipedia abstracts. Then, we converted the XML format into $\mathrm{JSON}^{6}$ format. After that we cleaned the text using regular expressions which includes removing special characters, remove incomplete or blank sentences and abstracts, text between brackets, etc. In table II, we show all the steps performed for the preprocessing of the dumps.

The total number of abstracts before cleaning was around $74 \mathrm{k}$ in the Simple Wikipedia dump. Since we had not yet cleaned the dump, we did not count the parallel Wikipedia abstracts. After cleaning the data, we extracted around $48.8 \mathrm{k}$ abstracts from Simple Wikipedia and in parallel we found around $27.5 \mathrm{k}$ abstracts in Wikipedia as some of the abstracts are found blank, incomplete or missing. The total number of sentences extracted from the cleaned Simple Wikipedia was around $87 \mathrm{k}$, including $968.2 \mathrm{k}$ tokens, with most of the abstracts including two sentences. In parallel, the total number of sentences extracted from the cleaned Wikipedia dump was around $39.2 \mathrm{k}$, including around $586.7 \mathrm{k}$ tokens. In table III, we show a comparison between the two dumps before and after cleaning.

In order to test our intuition with respect to rewriting simplified text represented in the Simple Wikipedia abstracts to a machine-oriented CNL, we decided to analyze the presence of CNLs features in both within text abstracts of Simplified Wikipedia and the corresponding Wikipedia abstracts, the purpose being to measure how similar the Simplified text

\footnotetext{
${ }^{6}$ Java Script Object Notation
} 
TABLE I: Comparison showing the overlap between CNL rules and Simplified Text rules

\begin{tabular}{|l|l|l|}
\hline Metric & Common CNL Rules & Simplified Text Rules \\
\hline Short sentences & should not exceed 20 tokens & $\begin{array}{l}\text { Keep sentences short and informa- } \\
\text { tive }\end{array}$ \\
\hline Use active voices & recommended & recommended \\
\hline Lexicon & $\begin{array}{l}\text { Use approved words from the Dic- } \\
\text { tionary (controlled lexicon) }\end{array}$ & Basic English Word-list \\
\hline Idioms & $\begin{array}{l}\text { Do not make noun clusters of more } \\
\text { than three nouns }\end{array}$ & Avoid idioms (multi-words) \\
\hline
\end{tabular}

TABLE II: Corpus collection and Pre-processing steps

\begin{tabular}{|l|l|l|}
\hline Metric & Simple Wikipedia & Wikipedia \\
\hline Corpus & $\begin{array}{l}\text { Abstracts of all articles (extracted } \\
\text { from the dump) }\end{array}$ & $\begin{array}{l}\text { Abstracts of parallel Simple } \\
\text { Wikipedia articles }\end{array}$ \\
\hline Format & XML dump converted to JSON format \\
\hline \multirow{5}{*}{ Pre-processing } & $\begin{array}{l}\text { Text cleansing using regular expressions (e.g. remove special charac- } \\
\text { ters, very short sentences, blank abstracts, text between brackets..etc) }\end{array}$ \\
\cline { 2 - 2 } & Split each abstract into sentences (i.e sentence segmentation) \\
\cline { 2 - 2 } & Split each sentence into tokens (i.e tokenization) \\
\cline { 2 - 2 } & $\begin{array}{l}\text { Run Part of Speech tagging (POS) using NLTK tagger using Penn } \\
\text { Treebank Tag Set [16] over each sentence and and create a list of } \\
\text { POS tags for each sentence in the corpus (POS structures list). }\end{array}$ \\
\hline
\end{tabular}

TABLE III: Comparison of Simple Wikipedia \& parallel Wikipedia abstracts

\begin{tabular}{|l|l|l|}
\hline Metric & Simple Wikipedia & Parallel Wikipedia \\
\hline $\begin{array}{l}\text { No. of abstracts before } \\
\text { cleaning }\end{array}$ & 74,067 & N/A \\
\hline $\begin{array}{l}\text { No. of abstracts after clean- } \\
\text { ing }\end{array}$ & 48,880 & 27,539 \\
\hline $\begin{array}{l}\text { Total No. of sentences in the } \\
\text { corpus }\end{array}$ & 87,088 & 39,252 \\
\hline $\begin{array}{l}\text { Total No. of tokens in the } \\
\text { corpus }\end{array}$ & 968,231 & 586,732 \\
\hline
\end{tabular}

to the common CNL features identified in [13]. In regard to the feasibility of rewriting Simplified Wikipedia sentences to a CNL, our first assumption is that the simplified text should be logically less ambiguous and less complex than standard Wikipedia unstructured text. Consequently, its linguistic properties will overlap significantly more with CNLs than unstructured text. So in order to measure this, we analyzed some of the measurable properties from [13] that should be present in the Simplified English text, given that it could be rewritten into CNL. As shown in table IV, the first metric is the length of sentences (number of tokens/sentence). In Simple Wikipedia we found that more than $90 \%$ of the sentences does not exceed the 20 tokens. On the other side, sentences from the parallel Wikipedia abstracts are usually exceeding this limit. Although the guidelines for writing Simple Wikipedia abstracts recommended the authors to avoid using passive voices, we found that $34 \%$ of the sentences did not follow this rule. On the other hand, $51 \%$ of the sentences in Wikipedia articles, are written using the passive voice. Gerunds are the words that are formed with verbs but act as nouns e.g go swimming, were found to be $6 \%$ in Simple Wikipedia sentences and $21 \%$ in Wikipedia sentences. CNLs usually use determiners before nouns, so our test found that the tags which preceded nouns in the Simple Wikipedia sentences are ranked as follows: 1) Determiners, 2) Noun Phrases, 3) Prepositions, 4) Adjectives, but in the Wikipedia sentences the list was different as follows 1) Nouns, 2) Noun Phrases, 3) Prepositions, 4) Determiners. Moreover, noun clusters are found in $4 \%$ of the Simple Wikipedia sentences and $8 \%$ in the Wikipedia sentences. Hence, based on the observations above, we can confirm our hypothesis that the linguistic properties of Simplified Wikipedia text overlap more with CNLs than unstructured text.

Based on the results above, we conducted a deeper analysis of the Simple Wikipedia POS structures which overlapped completely with the CNL rules. This meant extracting all abstracts which follow the common CNLs rules in table IV from the original dataset dump, excluding the remainder. As shown in table $\mathrm{V}$, the total number of abstracts from the Simple Wikipedia dump that follow the CNL rules in Table IV are found to be around $20.4 \mathrm{k}$. These abstracts include around $36.5 \mathrm{k}$ sentences, with $383.5 \mathrm{k}$ tokens. The result is our 
TABLE IV: Results of analysing the CNL properties across Simple Wikipedia and Wikipedia sentences

\begin{tabular}{|l|l|l|}
\hline Metric & Simple Wikipedia & Parallel Wikipedia \\
\hline Maximum Tokens/sentence $\leq 20$ & Yes & No \\
\hline Passive voices & $34 \%$ & $51 \%$ \\
\hline Gerunds & $6 \%$ & $21 \%$ \\
\hline Articles preceding nouns & DT,NNP,IN,JJ & NN,NP,IN,DT \\
\hline Noun clusters & $4 \%$ & $8 \%$ \\
\hline
\end{tabular}

TABLE V: Results of extracting the Simple Wikipedia abstracts that follow the CNL rules.

\begin{tabular}{|l|l|l|}
\hline Metric & Result & $\begin{array}{l}\text { Percentage from the total } \\
\text { corpus }\end{array}$ \\
\hline $\begin{array}{l}\text { No. of abstracts that are fully overlapping } \\
\text { with the common CNL properties. }\end{array}$ & 20,647 & $42.2 \%$ \\
\hline Total No. of Sentences & 36,560 & $42 \%$ \\
\hline Total No. of Tokens & 383,555 & $39.6 \%$ \\
\hline
\end{tabular}

reference corpus for our remaining experiments.

\section{RESULTS}

Since Simplified English rules and style guides request authors to use preferred sentence forms such as Subject-Verb-Directobect, and Subject-Verb-IndirectObject ${ }^{7}$ ), our second hypothesis is that most of the sentences, which have the same POS tags structure/pattern (forms), can be rewritten into CNL using a few rules without changing their semantics. So, we created a dictionary that includes the POS tags structures of all the sentences in the corpus. The main aim of creating this dictionary is to discover to which extent the authors of the Simple Wikipedia abstracts followed the guidelines of writing preferred sentence structures for writing the simplified text. So, we grouped all similar POS tags structures together and count them, in order to estimate the percentage of unique POS tags structures and the percentage of repeated POS structures in the corpus. This would thus help us approximate the number of rewrite rules needed to map Simplified Wikipedia sentences into CNL. We found total number of 22,083 unique POS tag structures. Next, we estimated the number of sentences that belongs to a group of POS tags structures. As shown from table VI, we present 5 cases from the dictionary. For example, the dictionary shows that 102 POS tags structures include around 7.8k sentences, and 629 POS tags structures include around 12.6k sentences. This analysis indicated that there are a lot of repeated POS structures that could be found in the corpus.

We extracted the top 5 repeated POS tags structures in the corpus and we show our analysis on them in table VII. We found that the first structure is found 634 times in the corpus. We experimented with rewriting a few sentences which match this POS structure into ACE. So, in order to rewrite this POS tags structure, we need to chunk the noun clusters into one noun, to be accepted by the APE parser and validated as an ACE sentence for automatic translation into DRS formalisms.

\footnotetext{
${ }^{7}$ https://simple.wikipedia.org/wiki/Wikipedia:How_to_write_Simple_ English_pages
}

Although POS tags structures number 2, 3 and 5 are not as frequent as POS tags structure number 1, they did not require any rewriting to be accepted by the ACE parser or converted into DRS as they tend to be very basic sentences. The POS tags structure number 4, can rewritten using a noun phrase chunker in the beginning of the sentence to chunk the names as one noun, and another noun phrase chunker in the end of the sentence to chunk the combination consists of a noun after an adjective into one noun. Although table VI shows that there are some repeated POS tags structures in the corpus, still the number of sentences to POS tags structures ratio $(12,630$ sentence /630 POS tags structure) is not high, if we compared it to the total size of the corpus which is around $36.5 \mathrm{k}$ sentences. This means that in order to rewrite 12,630 sentences into CNL we need to implement rules that can cover the 630 different POS tags structures, which is a lot of rules that will lead to rewriting only $35 \%$ of the corpus. Although it was shown from table VII that some few basic sentences did not require a rule for rewriting these sentences, the table showed that the percentage of these is still very low.

\section{A. Annotation and Validation}

So, in order to overcome this problem we found from our analysis that a common rule from table VII contains noun clusters and/or adjective noun clusters - noun phrase chunks effectively. So, we applied chunking rules on all the sentences in the corpus and assigned them a new POS tag COMP. A new set of new POS tags structures are created and the results from this analysis is summarized in table VIII. In the table we notice that around 19.2k sentences are captured together under 300 POS tags structures only, out of total 13,867 POS tags structures. This means that if we can rewrite the 300 structures into ACE CNL, we envisage having around 19.2k sentences in ACE CNL format and consequently into the DRS formal representation and exported to OWL.

Open further analysis after extracting from the corpus the $19.2 \mathrm{k}$ sentences that belong to the 300 POS tags structures, 
TABLE VI: Grouping sentences that belong to the same POS tags structure

\begin{tabular}{|l|l|l|l|}
\hline $\begin{array}{l}\text { No. of POS tags } \\
\text { Structures }\end{array}$ & $\begin{array}{l}\text { Percentage from the total } \\
\text { No. of POS tags Structures }\end{array}$ & No. of sentences & $\begin{array}{l}\text { Percentage from the } \\
\text { total No. of sen- } \\
\text { tences }\end{array}$ \\
\hline 102 & $0.4 \%$ & 7,809 & $21.3 \%$ \\
\hline 115 & $0.5 \%$ & 8,080 & $22.1 \%$ \\
\hline 182 & $0.8 \%$ & 9,203 & $25.1 \%$ \\
\hline 289 & $1.3 \%$ & 10,460 & $28.6 \%$ \\
\hline 629 & $2.8 \%$ & 12,630 & $34.5 \%$ \\
\hline
\end{tabular}

TABLE VII: A table showing the sentences that belong to the top 5 dominating POS tags structures after rewriting into a CNL.

\begin{tabular}{|l|l|l|l|l|}
\hline Rank & POS Pattern/Structure & Count Examples & ACE CNL \\
\hline 1 & $\begin{array}{l}\text { NNP VBZ DT NN IN NNP IN } \\
\text { DT NNP NNPS }\end{array}$ & 634 & $\begin{array}{l}\text { - Macon is a city of Illinois } \\
\text { in the United States. } \\
\text { - Agency is a city of Iowa } \\
\text { in the United States }\end{array}$ & $\begin{array}{l}\text { - Macon is a city of Illinois } \\
\text { in the n:United-States. } \\
\text { - Agency is a city of Iowa } \\
\text { in the n:United-States. }\end{array}$ \\
\hline 2 & NNP VBZ DT NN IN NNP & 295 & $\begin{array}{l}\text { - Aalborg is a city in Den- } \\
\text { mark } \\
\text { - Helene is a moon of Sat- } \\
\text { urn }\end{array}$ & $\begin{array}{l}\text { - Aalborg is a city in Den- } \\
\text { mark. } \\
\text { - Helene is a moon of Sat- } \\
\text { urn. }\end{array}$ \\
\hline 3 & NNP VBZ DT NN & 199 & $\begin{array}{l}\text { - Thirteen is a number } \\
\text { - Waitby has a castle }\end{array}$ & $\begin{array}{l}\text { - Thirteen is a number } \\
\text { - Waitby has a castle }\end{array}$ \\
\hline 5 & NNP NNP NNP VBD DT JJ & 196 & $\begin{array}{l}\text { - Guy Henry Ourisson was } \\
\text { a French chemist. } \\
\text { Edna May Oliver was an } \\
\text { American actress }\end{array}$ & $\begin{array}{l}\text { - Guy-Henry-Ourisson is a } \\
\text { n:French chemist. } \\
\text { Edna-May-Oliver is an } \\
\text { n:American-actress. }\end{array}$ \\
\hline
\end{tabular}

TABLE VIII: Grouping sentences that belong to the same POS tags structure after chunking nouns and adjectives.

\begin{tabular}{|l|l|l|l|}
\hline $\begin{array}{l}\text { No. of POS tags } \\
\text { Structures }\end{array}$ & $\begin{array}{l}\text { Percentage from the total } \\
\text { No. of POS tags Structures }\end{array}$ & No. of sentences & $\begin{array}{l}\text { Percentage from the } \\
\text { total No. of sen- } \\
\text { tences }\end{array}$ \\
\hline 300 & $2.1 \%$ & 19,236 & $52.6 \%$ \\
\hline 605 & $4.3 \%$ & 21,203 & $58 \%$ \\
\hline
\end{tabular}

we applied a total of 10 rules $^{8}$ ) to the sentences, where some of the results of these rules can be shown in table IX. The table shows the top 5 POS tags structures after the chunking took place. The highest occurring POS tags structure was found 2664 times. The rewriting rule for this structure is to chunk the nouns at the end of the sentence. The second most dominating structure was found 1399 times, and the rewriting rule chunked the nouns at the beginning and of the sentence. Structure number 3 occurred 1088 times, and the rewriting rule chunked the nouns at the end of the sentence. Structure

\footnotetext{
${ }^{8}$ https://drive.google.com/open?id=1eBUXZ8tESIML3jEptqG4aci4-_ BmODkP
}

number 4 occurred 926 times and the rewriting rule chunked a noun/group of nouns, followed by POS tag IN, followed by a noun/group of nouns. The last structure occurred 885 times, where the past verb of the sentence is rewritten into the present tense and the nouns at the beginning and end of the sentence are chunked.

\section{B. Evaluation}

The evaluation of the generated resource is performed on two sides, 1) The system coverage, and 2) The Semantic similarity of the mapped sentences. Since we developed syntactic rules to cover the top 300 POS tags structures, the system coverage is to estimate how many sentences the system was 
TABLE IX: A table showing the sentences that belong to the top 5 dominating POS tags structures (using chunking) after rewriting into a CNL.

\begin{tabular}{|c|c|c|c|c|}
\hline \multicolumn{2}{|c|}{ Rank POS Pattern/Structure } & \multicolumn{2}{|c|}{ Count Examples } & ACE CNL \\
\hline 1 & $\begin{array}{l}\text { COMP VBZ DT COMP IN } \\
\text { COMP }\end{array}$ & 2664 & $\begin{array}{l}\text { - Alkmene is a person in Greek } \\
\text { mythology. } \\
\text { - Anyang is a city in South Korea. }\end{array}$ & $\begin{array}{l}\text { - Alkmene is a person in the n:Greek mythol- } \\
\text { ogy. } \\
\text { - Anyang is a city in the n:South-Korea. }\end{array}$ \\
\hline 2 & COMP VBZ DT COMP & 1399 & $\begin{array}{l}\text { - Alicia Bridges is an American singer. } \\
\text { - Blood transfusion is a medical term. }\end{array}$ & $\begin{array}{l}\text { - Alicia-Bridges is an n:American-singer. } \\
\text { - Blood-transfusion is a n:medical-term. }\end{array}$ \\
\hline 3 & $\begin{array}{l}\text { COMP VBZ DT COMP IN } \\
\text { COMP IN DT COMP }\end{array}$ & 1088 & $\begin{array}{l}\text { - Gilbert is a city of Iowa in the United } \\
\text { States. } \\
\text { - Iphiclides is a genus of Butterflies in } \\
\text { the family Papilionidae. }\end{array}$ & $\begin{array}{l}\text { - Gilbert is a city of Iowa in the n:United- } \\
\text { States. } \\
\text { - Iphiclides is a genus of Butterflies in the } \\
\text { n:family-Papilionidae. }\end{array}$ \\
\hline 4 & $\begin{array}{l}\text { COMP VBZ DT COMP IN } \\
\text { DT COMP IN COMP }\end{array}$ & 926 & $\begin{array}{l}\text { - Anhui is a province in the east of } \\
\text { China. } \\
\text { - Agriculture is an important part of } \\
\text { the economy of Azerbaijan. }\end{array}$ & $\begin{array}{l}\text { - Anhui is a province in the n:east-of-China. } \\
\text { - Agriculture is an important part of the } \\
\text { n:economy-of-Azerbaijan. }\end{array}$ \\
\hline 5 & COMP VBD DT COMP & 885 & $\begin{array}{l}\text { - Abel Ricardo Laudonio was an Ar- } \\
\text { gentine boxer. } \\
\text { - Braniff International Airways was an } \\
\text { American airline. }\end{array}$ & $\begin{array}{l}\text { - Abel-Ricardo-Laudonio is an } \mathrm{n} \text { :Argentine- } \\
\text { boxer. } \\
\text { - Braniff-International-Airways is an } \mathrm{n} \text { :Ame } \\
\text { rican-airline. }\end{array}$ \\
\hline
\end{tabular}

TABLE X: Evaluation of the coverage of the rewriting system.

\begin{tabular}{|l|l|l|}
\hline Metric & Total No. of sentences & Coverage \\
\hline Rewritten into ACE CNL & 17,199 & $89.4 \%$ \\
\hline Not rewritten into ACE CNL & 2,037 & $10.6 \%$ \\
\hline
\end{tabular}

able to rewrite out of the whole corpus. After we applied the rules on the corpus, we can see from Table $\mathrm{X}$ that we were able to rewrite 17,199 sentences out of 19,236 sentences to the ACE CNL. Thus, the system coverage is equal to $89.4 \%$, and sentences that failed to parse are 2037 sentences that represent $10.6 \%$ of the corpus. These sentences failed to parse as the developed rules failed to rewrite them into an interpretable ACE CNL structure, thus they were refused by the APE parser. The rewrite rules are developed for the most repeated POS structures to rewrite as much sentences as possible, neglecting individual POS structures. All the rewritten sentences are passed to the APE parser web service and validated for being successfully parsed.

The second evaluation is done to ensure that the semantics has not changed after the rewriting happened, we need to test whether the generated ACE CNL sentence preserved the same semantics of its mapped SE sentence. So, we extracted a representative sample from the corpus after estimating the sample size from [18] and it was found to be 527 sentences. Then, we computed the semantic textual similarity between the SE sentence and its mapped ACE CNL sentence based on the research in [19]. The result showed that our system preserves the semantic similarity between all the SE sentences, and their mapped ACE CNL sentences in the sample set.
The resource is available for download ${ }^{9}$ ) and includes all the 17,199 sentences from original Simple Wikipedia and their parallel ACE rewritten sentences. These ACE sentences should be a very valuable resource to the community for exploitation in different applications related to NLP for knowledge base population.

\section{CONCLUSION}

We have presented a linguistic resource that is both humanreadable and semantically machine interpretable. To our knowledge this resource is the first aligned dataset across a human-oriented and machine-oriented CNL as well as unstructured text. The dataset is well represented in that it takes almost the entire Simplified Wikipedia abstract population postcleansing. We have provided corpus statistics and linguistics analysis, which have confirmed our hypotheses with respect to rewriting Simplified English to ACE and its subsequent transformation into logical and knowledge representations such as DRS and OWL respectively.

This resource could be exploited in other fields beyond CNLs for knowledge based population. Potential applications include generating general knowledge for expert and knowledge based systems and ontology aware NLP applications

\footnotetext{
${ }^{9} \mathrm{https} / / /$ drive.google.com/open?id=1eBUXZ8tESIML3jEptqG4aci4-_ BmODkP
} 
as well as knowledge based MT, automated reasoning, language learning as well as teaching and learning resources for knowledge engineering and logic programming. In addition to, generalization to other languages can be done using present frameworks such as GF to translate ACE to other languages [20].

Future work with respect to this resources will involve augmenting the aligned human to machine-oriented CNL content with semantic metadata such as $\mathrm{RDF}^{10}$ generated from ACE2OWL ${ }^{11}$ [17]. Other work will involve additional corpus analysis and rule generation for rewriting less common Simplified English POS patterns.

\section{ACKNOWLEDGMENT}

This work has emanated from research supported in part by a research grant from Science Foundation Ireland (SFI) under Grant Number SFI/12/RC/2289 and in part by the SSIX Horizon 2020 project (grant agreement No 645425).

\section{REFERENCES}

[1] Hazem Safwat and Brian Davis. 2014. A brief state of the art of CNLs for ontology authoring. International Workshop on Controlled Natural Language. pp. 190-200. Springer.

[2] Tobias Kuhn. 2014. A survey and classification of controlled natural languages. Computational Linguistics 40, pp. 121-170.

[3] Hujisen WO. 1998. Controlled language: an introduction. Second International Workshop on Controlled Language Applications (CLAW), Pittsburgh.

[4] Wojcik, Richard H., Heather Holmback, and James Hoard. 1998. Boeing Technical English: An extension of AECMA SE beyond the aircraft maintenance domain. Second International Workshop on Controlled Language Applications (CLAW), Pittsburgh.

[5] Ogden, Charles Kay. 1944. Basic English: A general introduction with rules and grammar. Vol. 29. K. Paul, Trench, Trubner.

[6] Hazem Safwat and Brian Davis. 2017. CNLs for the semantic web: a state of the art. Language Resources and Evaluation, 51(1), pp.191-220.

[7] Fuchs, Norbert E., Kaarel Kaljurand, and Tobias Kuhn. 2008. Attempto controlled english for knowledge representation. Reasoning Web (pp. 104-124). Springer, Berlin, Heidelberg.

[8] Xu, Wei, Chris Callison-Burch, and Courtney Napoles. 2015. Problems in current text simplification research: New data can help. Transactions of the Association of Computational Linguistics, 3(1), pp.283-297.

[9] Woodsend, Kristian, and Mirella Lapata. 2017. Text rewriting improves semantic role labeling. Journal of Artificial Intelligence Research, 51, pp.133-164.

[10] Basile, Valerio, Johan Bos, Kilian Evang, and Noortje Venhuizen. 2012. Developing a large semantically annotated corpus. Eighth International Conference on Language Resources and Evaluation.

[11] Martha Palmer, Paul Kingsbury, and Daniel Gildea. 2005. The proposition bank: An annotated corpus of semantic roles. Computational Linguistics, 31(1):71106.

[12] Collin F. Baker, Charles J. Fillmore, and John B. Lowe. 1998. The Berkeley FrameNet project. 17th International Conference on Computational Linguistics.

[13] OBrien, Sharon. 2003. Controlling controlled english. an analysis of several controlled language rule sets. Proceedings of EAMT-CLAW, 3, pp.105-114.

[14] Bird, Steven, and Edward Loper. 2004. Text rewriting improves semantic role labeling. Journal of Artificial Intelligence Research, 51, pp.133-164.

[15] Marcus, Mitchell P., Mary Ann Marcinkiewicz, and Beatrice Santorini. 1998. Building a large annotated corpus of English: The Penn Treebank.. Computational linguistics, 19(2), pp.313-330.

[16] Fuchs, Norbert E., Kaarel Kaljurand, and Tobias Kuhn. 2008. Discourse representation structures for ACE 6.0. Department of Informatics.

${ }^{10}$ https://www.w3.org/RDF/

${ }^{11}$ https://www.w3.org/2001/sw/wiki/ACE
[17] Kaljurand, Kaarel, and Norbert E. Fuchs. 2006. Mapping Attempto Controlled English to OWL DL. 3rd European Semantic Web Conference. Demo and Poster Session, Budva, Montenegro.

[18] Smith, S. 2013. Determining sample size: How to ensure you get the correct sample size. E-Book (c) Qualtrics Online Sample.

[19] Han, L., Kashyap, A.L., Finin, T., Mayfield, J. and Weese, J. 2013. UMBC_EBIQUITY-CORE: semantic textual similarity systems. In Second Joint Conference on Lexical and Computational Semantics (SEM), Volume 1: Proceedings of the Main Conference and the Shared Task: Semantic Textual Similarity (Vol. 1, pp. 44-52).

[20] Kaljurand, K. and Kuhn, T. 2013. A multilingual semantic wiki based on Attempto Controlled English and Grammatical Framework. In Extended Semantic Web Conference (pp. 427-441). Springer, Berlin, Heidelberg. 